Review Article

\title{
Role of Extracellular Vesicles in Placental Inflammation and Local Immune Balance
}

\author{
Zengfang Wang, ${ }^{1}$ Ruizhen Yang, ${ }^{2}$ Jiaojiao Zhang, ${ }^{3}$ Pingping Wang, ${ }^{1}$ Zengyan Wang, \\ Jian Gao $\left(\mathbb{D},{ }^{4}\right.$ and Xue Liu ${ }^{2}{ }^{2}$ \\ ${ }^{1}$ Department of Gynecology and Obstetrics, Maternal and Child Health Hospital of Weifang Medical University, \\ Weifang 261000, China \\ ${ }^{2}$ Operating Room, Zhucheng People's Hospital, Zhucheng 262200, China \\ ${ }^{3}$ Central Laboratory of Weifang People's Hospital, Weifang 261000, China \\ ${ }^{4}$ Department of Paediatrics, Maternal and Child Health Care Hospital of Weifang, Weifang 261000, China
}

Correspondence should be addressed to Jian Gao; gaojian1650@126.com and Xue Liu; rmyyliuxue@163.com

Received 7 February 2021; Revised 26 April 2021; Accepted 25 May 2021; Published 19 June 2021

Academic Editor: Bingjie Gu

Copyright $\odot 2021$ Zengfang Wang et al. This is an open access article distributed under the Creative Commons Attribution License, which permits unrestricted use, distribution, and reproduction in any medium, provided the original work is properly cited.

Background. Pregnancy maintenance depends on the formation of normal placentas accompanied by trophoblast invasion and vascular remodeling. Various types of cells, such as trophoblasts, endothelial cells, immune cells, mesenchymal stem cells (MSCs), and adipocytes, mediate cell-to-cell interactions through soluble factors to maintain normal placental development. Extracellular vesicles (EVs) are diverse nanosized to microsized membrane-bound particles released from various cells. EVs contain tens to thousands of different RNA, proteins, small molecules, DNA fragments, and bioactive lipids. EV-derived microRNAs (miRNAs) and proteins regulate inflammation and trophoblast invasion in the placental microenvironment. Maternal-fetal communication through EV can regulate the key signaling pathways involved in pregnancy maintenance, from implantation to immune regulation. Therefore, EVs and the encapsulating factors play important roles in pregnancy, some of which might be potential biomarkers. Conclusion. In this review, we have summarized published studies about the EVs in the placentation and pregnancy-related diseases. By summarizing the role of EVs and their delivering active molecules in pregnancy-related diseases, it provides novel insight into the diagnosis and treatment of diseases.

\section{Introduction}

Extracellular vesicles (EVs) are secreted by cell membrane or cells with diameters ranging from $40 \mathrm{~nm}$ to $1000 \mathrm{~nm}[1,2]$. EVs mainly include microbubbles, ectosomes, exosomes, and microparticles according to their subcellular sources [3-5]. Exosomes with a diameter of $40 \sim 100 \mathrm{~nm}$ are the most commonly studied EVs, which are firstly found in sheep reticulocytes [6]. EV plays an important role in a series of biological processes by transferring lipids, proteins, nucleotides, and other bioactive components. These encapsulated bioactive molecules can be delivered to specific targeted cells (Figure 1). In particular, some EVs carrying noncoding RNAs, such as microRNA (miRNA) and long-chain noncoding RNAs (lncRNA), can be transferred to specific cells and regulate the expression and function of target mRNA at different biological stages [7]. EVs participate in the exchange of substances and information between cells mainly through the following pathways [8-10]. Firstly, EV membrane proteins can bind to the targeted cell membrane, thereby activating the signaling pathway in cells. Secondly, EVs can be digested by proteases in extracellular matrix. The digested fragments can be used as ligands to bind to the receptors on the cell membrane, thereby regulating intracellular communication. Thirdly, EVs can be directly fused with the target cell membrane, and then, EVs containing bioactive components are nonselectively released into recipient cells.

EVs from different cell sources can exert multiple effects. EVs derived from mesenchymal stem cells (MSC) can significantly improve myocardial cell survival, prevent myocardial 


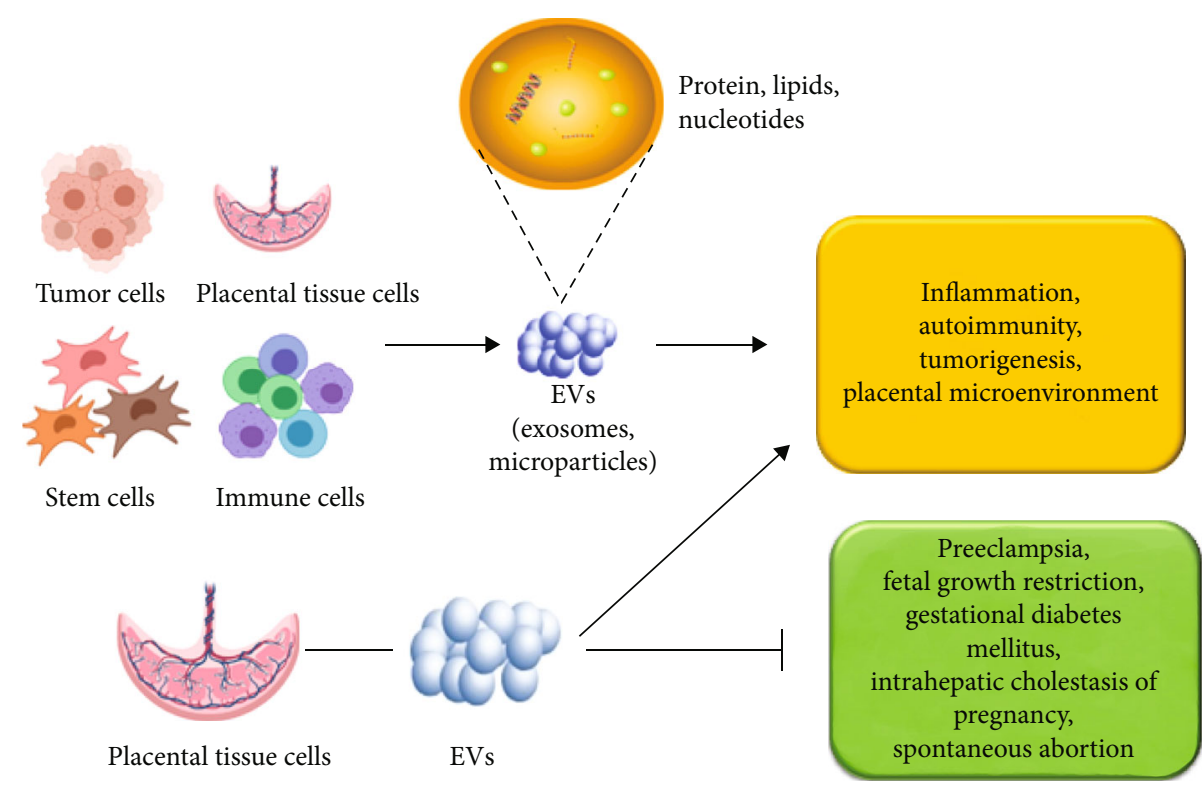

FIGURE 1: Role of EVs in placenta and pregnancy disorders. EVs mainly include exosomes and particles, which can be derived from stem cells, tumor cells, immune cells, and placental tissue cells. They participate in intercellular communication by transmitting bioactive proteins, lipids, and nucleotides to targeted cells. EVs, especially EVs derived from placental tissue cells, play a crucial role in regulating inflammation, autoimmune, tumor occurrence, and placental microenvironment balance. EVs have been used to prevent pregnancy diseases such as PE, fetal growth restriction, and spontaneous abortion.

cell injury, promote angiogenesis, and improve cardiac function by regulating inflammation and autoimmune [11-13]. Due to the advantages of nanomolecular structure and excellent biocompatibility, EVs have great application potential as drug carriers. MSC-derived EVs are used to treat chronic skin ulcers, which can promote wound healing and reduce scar formation [14]. In addition, EVs play a key role in tumor occurrence, immune surveillance, immune escape, and tumor microenvironment reprogramming [15-17]. Accordingly, EVs and their encapsulated bioactive molecules are essential for intercellular communication in many diseases, including pregnancy disorders. The role of EVs in immune regulation has been extensively studied. EVs from intestinal epithelial cells (IECs) released during sepsis have been reported to improve intestinal inflammation by transferring miRNAs into cells [18]. EVs are involved in maintaining $\mathrm{T}$ cell tolerance and long-term $\mathrm{T}$ cell memory [19]. EVencapsulated proteins participate in immune regulation by influencing the secretion of anti-inflammatory cytokines [20]. Thyroid stimulating hormone receptor (TSHR) released by EV improves autoantibody-mediated activation of Graves disease by blocking autoantibodies [21]. Baskaran et al. reported that EVs play an indispensable role in sperm maturation by regulating autoimmunity [22]. This local immunity privilege at the maternal-fetal interface has been attributed to the expression of Fas ligand (FasL) related to placental exosomes, programmed death ligand 1 (PD-L1), and TNFrelated apoptosis-inducing ligand (TRAIL), which all induce maternal T cell incompetence and death $[23,24]$. Expression of NKG2D receptor ligand, UL-16-binding protein (ULBP), and MHC class I chain-related protein (MIC) on placental exosomes have been shown to downregulate NK cell activity and inhibit maternal cytotoxicity [25].
In the past decades, the role of EV in regulating placenta implantation and pregnancy diseases has attracted much attention due to its key role in regulating inflammation and immunity. The aim of this study is to summarize the current published studies on the association of EVs with placenta implantation and pregnancy-related diseases. In particular, we aim to explore the role of EVs in the diagnosis and treatment of pregnancy-related diseases.

\section{EVS from Placental Tissues and Stem Cells}

Placenta is the fundamental physiological barrier for maternal immune tolerance to fetus and various pathogens. EVs released by syncytiotrophoblasts, trophoblasts, extravillous trophoblasts, and placental vascular endothelial cells play a key role in pregnancy [26]. Placenta and umbilical cord are rich in MSCs, which are promising treatment strategies for various diseases [27]. It has been shown that EVs from human umbilical cord MSCs (hUC-MSCs) could protect against severe burn-induced hyperinflammation through the way of paracrine secretion [28]. Surico et al. have found that hUC-MSCs are involved in preeclampsia (PE) and affect fetal growth restriction (FGR) by delivering bioactive components encapsulated in EVs [29]. hUC-MSC-derived EVs have also been found to confer effect on the paracrine of trophoblast cells, which facilitates their use in treating pregnancyrelated disorders. Decidual MSC-derived EVs can also exert inhibitory effects on angiogenesis and maintain the balance of maternal-fetal interface by regulating macrophage polarization in PE [30]. Accordingly, placenta tissue MSCs and hUC-MSC-derived EVs play key roles in maintaining placental functions and protecting against pregnancy disorders. 
Accumulated data have shown that MSC transplantation may be extremely beneficial for pregnancy-associated diseases [31]. In the past few years, the use of MSC-EVs in pregnancy disorders has drawn more and more attention. Placental tissue cell-derived EVs can be produced regardless of physiological or pathological pregnancy. Reduced circulating EVs are associated with placental dysfunction [32]. Circulating EVs play a crucial role during pregnancy [33]. EVs derived from placental tissue cells increased gradually with the progress of pregnancy, especially during delivery, but significantly decreased to nonpregnancy level 48 hours after delivery [34]. EVs derived from placental tissue cells can selectively transfer bioactive molecules, such as stressinduced protein molecules, apoptosis-related molecules, cytokines, mRNA, and miRNA. EVs actively participate in maternal-fetal communication during pregnancy by regulating different processes [35]. The fetus is the mother's allogeneic graft. Successful pregnancy depends on the maintenance of maternal-fetal interface immune tolerance. Th1/Th2 balance during pregnancy is essential to maintain maternal and fetal microenvironment [36]. Placenta-derived EVs promote maternal immune tolerance by inhibiting maternal $\mathrm{T}$ cell response and maintaining Th1/Th2 balance [35]. Placenta-derived EVs produce Syn-2, which proves to play an immunosuppressive role in placenta [37]. Placental tissue-derived EVs and exosome inclusion factors are important regulators of placental immunity and homeostasis [37]. The delivery of bioactive molecules by EVs is beneficial to placental function. During pregnancy, EVs can be transported to the fetal side as drugs and other goods [38]. EVs also play an important role in regulating systemic inflammation by releasing cytokines. Therefore, EVs from placenta tissues and stem cells are essential for maintaining maternalfetal immunity and protecting inflammation.

MSC-derived noncoding RNAs can serve as useful biomarkers in pregnancy disorders [28-30]. However, whether MSC-derived noncoding RNAs can exert their effects through EVs and mediate intercellular communications in maternal-fetal immunity needs to be elucidated in the future. During the past few decades, noncoding RNAs derived from placental stem cells have been demonstrated to participate in pregnancy regulation, such as lncRNAs and miRNAs [3941]. Placental tissue-derived EV miRNAs have been implicated in the antiviral immunity at the maternal and fetal interface [42]. In addition, studies have found that EVs from placental tissue cells also have significant effects on pregnancy complications by transferring miRNAs to recipient cells [43]. Accordingly, those noncoding RNAs from placental tissues and stem cell-derived EVs serve as key biomarkers for gestational disorders.

\section{EVS and Pregnant Disorders}

EVs are the "fingerprints" of their primitive cells and are involved in regulating pregnancy complications. EVs derived from placental tissue may be another way for the fetus to communicate with mothers. EV regulates inflammatory cascade reactions in some complex pregnancy, such as PE and intrauterine growth restriction [44]. Here, we summarize the research progress of the role of EVs and their encapsulated factors in some common pregnancy diseases (Table 1).

3.1. EVS and PE. PE is an idiopathic pregnancy disease characterized by hypertension and proteinuria after 20 weeks of pregnancy. The global incidence of $\mathrm{PE}$ is $3-8 \%$, and its pathogenesis is still unclear. The basic pathological changes of PE are systemic vasospasm, vascular endothelial injury, and ischemia. PE leads to reduced perfusion of all organs and poses risks to both mothers and children. Its performance is often significantly improved after childbirth or termination of pregnancy, suggesting that placental-derived media play a key role in the pathogenesis of PE.

A number of studies have suggested EVs and their encapsulated factors are involved in the pathogenesis of PE [45, 46]. Bioactive molecules in placental tissue-derived EVs might be as potential biomarkers for the diagnosis and prognosis of PE [47]. Placental protein 13 (PP13) has been identified in maternal circulating exosomes or microvesicles [48]. Low serum level of PP13 predicts high risk of PE and other obstetric complication [48]. Salomon et al. have found that total plasma exosomes and placental tissue exosomes both significantly increased in patients with PE compared with normal pregnancies [49]. Similarly, placental EVs in the blood circulation of PE patients are significantly lower than that in patients with early-onset PE [50]. Chang et al. showed that exosome soluble fms-like tyrosine kinase-1 (sFlt-1) and soluble endoglin (sEng) inhibited the growth and tube formation of human umbilical vein endothelial cells in PE [51]. In addition, circulating EVs from placenta can activate immune cells and may affect the production of inflammatory cytokines in PE pregnancy [52]. As a result, $\mathrm{EV}$-delivering factors are essential regulators in $\mathrm{PE}$.

In the last decade, EV-encapsulated noncoding RNAs have been found to affect the development of PE [40]. Some placental tissue-derived miRNAs can be released into the maternal circulation from the trophoblast layer via EVs [53]. A few EV-encapsulated miRNAs are potential markers for PE [54]. Diverse miRNA profiles in circulating EVs from early and late onset of PE have been demonstrated, which implicates that EV-derived miRNAs are key factors associated with PE [55]. Li et al. have demonstrated there are specific miRNA expression and signals in serum exosomes from PE pregnancies, suggesting the modifying effect of plasma exosomal miRNAs in the pathogenesis of PE [56]. Increased expression of miR-201-3p has been demonstrated in the circulating EVs of patients with PE [57]. The study by Shen et al. has suggested that placental tissue cellderived exosomal miR-155 inhibits the expression of endothelial nitric oxide synthase in PE [58]. In addition, there are many other EV-derived miRNAs demonstrated as key biomarkers in PE, such as EV-encapsulated miR-136 and miR-495 [59]. Exosomal miR-210 has been reported to be produced by trophoblasts and participates in the intercellular communication in PE [60]. Some EV-derived miRNAs have been suggested as circulating markers for PE, including miR517-5p, miR-520a-5p, and miR-525-5p [61]. Accordingly, EV-encapsulated noncoding RNAs are key regulators in PE. 
TABLE 1: EV-derived biomarkers in autoimmunity and pregnancy disorders.

\begin{tabular}{|c|c|c|c|c|c|}
\hline Biomarker & Type of biomarker & Type of derived EVs & $\begin{array}{l}\text { Diseases and } \\
\text { pregnancy } \\
\text { condition }\end{array}$ & Function & Reference \\
\hline Thyrotropin receptor & Receptor & Exosomes & $\begin{array}{l}\text { Graves' } \\
\text { disease }\end{array}$ & $\begin{array}{c}\text { Sequestering autoantibody and } \\
\text { ameliorating autoantibody-mediated } \\
\text { activation }\end{array}$ & {$[21]$} \\
\hline $\begin{array}{l}\text { UL-16-binding } \\
\text { protein }\end{array}$ & Protein & Exosomes & $\begin{array}{l}\text { Placenta } \\
\text { during } \\
\text { pregnancy }\end{array}$ & $\begin{array}{l}\text { Downregulate NK cell activity and } \\
\text { inhibit maternal cytotoxicity }\end{array}$ & {$[25]$} \\
\hline $\begin{array}{l}\text { MHC class I chain- } \\
\text { related protein }\end{array}$ & Protein & Exosomes & $\begin{array}{l}\text { Placenta } \\
\text { during } \\
\text { pregnancy }\end{array}$ & $\begin{array}{l}\text { Downregulate NK cell activity and } \\
\text { inhibit maternal cytotoxicity }\end{array}$ & {$[25]$} \\
\hline Syncytin-2 & Protein & Exosomes & $\begin{array}{l}\text { Normal } \\
\text { pregnancy }\end{array}$ & $\begin{array}{c}\text { Exerting immunosuppressive effects on } \\
\text { T cells }\end{array}$ & {$[37]$} \\
\hline Placental protein 13 & Protein & $\begin{array}{l}\text { Exosomes or } \\
\text { microvesicles }\end{array}$ & $\begin{array}{l}\text { PE and other } \\
\text { obstetric } \\
\text { complications }\end{array}$ & $\begin{array}{l}\text { Binding to glycosylated receptors, } \\
\text { bringing about hemagglutination, } \\
\text { immunoregulation and vasodilation }\end{array}$ & {$[48]$} \\
\hline $\begin{array}{l}\text { Soluble fms-like } \\
\text { tyrosine kinase-1 }\end{array}$ & Protein & Exosomes & $\mathrm{PE}$ & $\begin{array}{l}\text { Attenuating the proliferation, } \\
\text { migration, and tube formation of } \\
\text { human umbilical vein endothelial cells }\end{array}$ & {$[51]$} \\
\hline Soluble endoglin & Protein & Exosomes & $\mathrm{PE}$ & $\begin{array}{l}\text { Inhibiting the growth and tube } \\
\text { formation of human umbilical vein } \\
\text { endothelial cells }\end{array}$ & {$[51]$} \\
\hline MiR-201-3p & $\begin{array}{l}\text { Nucleotide/noncoding } \\
\text { RNA }\end{array}$ & Exosomes & $\mathrm{PE}$ & $\begin{array}{c}\text { Having a role in the pathomechanism } \\
\text { of PE }\end{array}$ & {$[57]$} \\
\hline MiR-155 & $\begin{array}{l}\text { Nucleotide/noncoding } \\
\text { RNA }\end{array}$ & Exosomes & $\mathrm{PE}$ & $\begin{array}{l}\text { Inhibiting the expression of endothelial } \\
\text { nitric oxide synthase }\end{array}$ & {$[58]$} \\
\hline MiR-136 and miR-495 & $\begin{array}{c}\text { Nucleotide/noncoding } \\
\text { RNA }\end{array}$ & $\begin{array}{l}\text { MSC-derived } \\
\text { exosomes }\end{array}$ & $\mathrm{PE}$ & $\begin{array}{l}\text { Promising circulating biomarkers in } \\
\text { early detection of PE }\end{array}$ & {$[59]$} \\
\hline MiR-210 & $\begin{array}{l}\text { Nucleotide/noncoding } \\
\text { RNA }\end{array}$ & Exosomes & $\mathrm{PE}$ & $\begin{array}{l}\text { Produced by trophoblasts and } \\
\text { participating in the intercellular } \\
\text { communication }\end{array}$ & {$[60]$} \\
\hline $\begin{array}{l}\text { Phosphoenolpyruvate } \\
\text { carboxykinase }\end{array}$ & Kinase & Urine exosomes & $\begin{array}{l}\text { Gestational } \\
\text { diabetes } \\
\text { mellitus }\end{array}$ & Affecting insulin resistance & {$[66]$} \\
\hline Sterile 20 -like kinase 1 & Protein & $\begin{array}{l}\text { Cardiac } \\
\text { microvascular } \\
\text { endothelial cell- } \\
\text { derived exosomes }\end{array}$ & Diabetes & $\begin{array}{l}\text { Inhibiting autophagy, promoting } \\
\text { apoptosis and suppressing glucose } \\
\text { metabolism }\end{array}$ & {$[70]$} \\
\hline $\begin{array}{l}\text { EVs-encapsulated } \\
\text { miRNAs }\end{array}$ & $\begin{array}{l}\text { Nucleotide/noncoding } \\
\text { RNA }\end{array}$ & EVs & $\begin{array}{l}\text { Gestational } \\
\text { diabetes } \\
\text { mellitus }\end{array}$ & $\begin{array}{l}\text { Serving as biomarkers for the diagnosis } \\
\text { and treatment of gestational diabetes } \\
\text { mellitus }\end{array}$ & [79-82] \\
\hline MiR-150 & $\begin{array}{c}\text { Nucleotide/noncoding } \\
\text { RNA }\end{array}$ & $\begin{array}{l}\text { Piglet's umbilical } \\
\text { vein-derived EVs }\end{array}$ & FGR & Promoting angiogenesis & {$[86]$} \\
\hline $\begin{array}{l}\text { MiR-21, miR-29a and } \\
\text { miR-590-3p }\end{array}$ & $\begin{array}{c}\text { Nucleotide/noncoding } \\
\text { RNA }\end{array}$ & Urinary exosomes & ICP & $\begin{array}{c}\text { Downregulating intercellular adhesion } \\
\text { molecule } 1\end{array}$ & {$[90]$} \\
\hline $\begin{array}{l}\text { MiR-300 and miR- } \\
299-5 p\end{array}$ & $\begin{array}{l}\text { Nucleotide/noncoding } \\
\text { RNA }\end{array}$ & EVs & $\begin{array}{l}\text { Congenital } \\
\text { obstructive } \\
\text { nephropathy }\end{array}$ & Reregulating renal fibrosis & [99] \\
\hline
\end{tabular}

EVs: extracellular vesicles; FGR: fetal growth restriction; ICP: intrahepatic cholestasis of pregnancy; MSCs: mesenchymal stem cells; PE: preeclampsia.

The role of stem cell EV-derived miRNAs in PE has also been extensively investigated in the past few years. It has been reported that the umbilical cord blood stem cells EV-derived miR-125a-5p plays a critical role in PE [62]. It has been demonstrated that hUC-MSC EVs can inhibit placental cell apo- ptosis and promote angiogenesis in PE [63]. Besides, MSCderived exosomal IncRNA H19 has been demonstrated to increase the invasion and migration of trophoblast cells by regulating let-7b through $\mathrm{AKT}$ signaling pathway in $\mathrm{PE}$ [64]. Accordingly, stem cell-derived EVs and the 
encapsulated bioactive molecules can regulate angiogenesis and autoimmune balance in $\mathrm{PE}$, which may be promising therapeutic strategy for patients with $\mathrm{PE}$.

3.2. EVS and Gestational Diabetes Mellitus. Pregnancy can cause diabetes in pregnant women who have no diabetes previously and also exacerbate the condition of patients with existing diabetes. Gestational diabetes has a greater impact on both mothers and children, and the near-term and longterm complications of mothers and children are higher. Pregnant women with diabetes are more likely to subject to hypertension during pregnancy than nondiabetic women [65]. Diabetes can increase the chance of infection, dystocia, birth canal injury, surgery, and postpartum bleeding in pregnant women as well as elevated incidence of large children, restricted fetal growth, spontaneous abortion, embryonic termination, and premature birth.

The development of gestational diabetes mellitus is related to heredity, autoimmunity, and environmental factors. Increasing evidence has suggested the crucial role of EVs in gestational diabetes mellitus. The study by Arias et al. has demonstrated the potential role of plasma EVencapsulated factors as early biomarkers for gestational diabetes mellitus [65]. Sharma et al. have found that human urine-derived EVs contained gluconeogenic enzymes that affect glucose metabolism [66]. Elevated phosphoenolpyruvate carboxykinase (PEPCK) in urine exosomes is related to high risk of diabetes and early insulin resistance [66]. In addition, currently available data has suggested EVs play an important role in autoimmune reaction in islets of type 1 diabetes [67]. Moreover, EVs produced by adipose tissue and muscle tissue regulate glucose and lipid balance and even the inflammatory environment by transferring specific molecules to a variety of insulin-sensitive peripheral tissues [67]. As a result, the effect of EVs in gestational diabetes mellitus is essential.

EV-encapsulated factors can be used as useful biomarkers for a variety of diseases and complications [68]. Many studies have shown EV-encapsulated proteins, mRNAs, and DNAs and are new diagnostic markers for diabetic nephropathy [68]. EVs can also be used as delivery vehicles for the treatment of diabetic nephropathy. A previous study has demonstrated that the urinary exosomal Elf3 may be an early noninvasive marker for podocyte injuries in diabetic nephropathy [69]. Mammalian sterile 20-like kinase 1- (Mst1-) enriched EVs released from cardiac microvascular endothelial cells play an important role in inhibiting autophagy, promoting apoptosis, and suppressing glucose metabolism in cardiomyocytes [70]. All these findings have supported the vital role of EVs in glucose metabolism and diabetes progression.

Insufficient myocardial angiogenesis can induce diabetesrelated ischemic cardiovascular [71]. CD133 ${ }^{+}$exosomes derived from human umbilical cord blood improve cardiac function in diabetic stroke mice [72]. MSC EVs protect $\beta$ cells from hypoxia-induced apoptosis [73]. More and more evidence suggests that EVs and delivered miRNAs have neuroprotective effects and may be a treatment for diabetesrelated stroke [74]. Studies have shown that EVs extracted from plasma of pregnant women with gestational diabetes significantly promote the production of inflammatory cytokines [75]. EVs can also regulate placental and fetal membrane endothelial dysfunction in gestational diabetes mellitus [76]. Adipose tissue-derived EVs mediate placental immunity in gestational diabetes mellitus, which may be associated with some adverse outcomes, including fetal overgrowth [77]. EVs represent a new mechanism for regulating maternal glucose homeostasis during pregnancy [78]. Taken together, the role of EVs in gestational diabetes mellitus is critical. At the same time, the mechanism of EVs wrapped bioactive factors in gestational diabetes remains to be further studied.

Increasing evidence has supported that EV-transferred miRNAs and other noncoding RNAs can be used as promising biomarkers for gestational diseases including gestational diabetes mellitus [79]. Nair et al. have reported that placental tissue-derived EV miRNAs can regulate skeletal muscle insulin sensitivity [80]. Placental tissue-derived EVs may exert effects on insulin sensitivity in normal and gestational diabetes mellitus pregnancies. Taken together, EVs offer new options for the treatment of gestational diabetes mellitus [81]. EV-encapsulated noncoding RNAs can also serve as biomarkers for the diagnosis and treatment of gestational diabetes mellitus [82].

3.3. EVS and Fetal Growth Restriction (FGR). FGR usually leads to low birthweight births. However, the underlying biological mechanism has not been fully elucidated. FGR can be secondary to various pregnancy complications, such as gestational hypertension, diabetes, and intrahepatic cholestasis. Previously found aberrantly expressed miRNAs in maternal circulation [83]. New evidence suggests that miRNAs are specifically expressed in maternal blood of FGR in the second trimester of pregnancy, suggesting possible fetal growth markers [84]. Besides, Miranda et al. reported that placental exosome-derived miRNAs in maternal plasma can predict fetal growth and can be used as indicators of placental function [85]. Studies have shown that EV miR-150 derived from umbilical vein of normal piglets can promote angiogenesis in intrauterine growth restriction pigs [86]. It is reported that circulating plasma exosomes in early pregnancy had the same C19MC microRNA as placental tissues of GH, PE, and FGR patients after delivery [87]. Maternal plasma exosome analysis of selected C19MC microRNAs [88] showed that FGR women had a new downregulated biomarker (miR-520a$5 p$ ) in the first three months of pregnancy, which was not found in maternal plasma analysis [89]. In summary, these established findings strongly demonstrate the important role of EVs and encapsulation factors including miRNAs in FGR, providing new clues for understanding the pathogenesis of FGR.

\subsection{EVS and Intrahepatic Cholestasis of Pregnancy (ICP). ICP} is a common complication during pregnancy. Due to bile acid toxicity, the incidence and mortality of perinatal complications increased significantly. The etiology of ICP is still unclear, which may be related to female hormones, genetic and environmental factors. The previously published data 
show the pivotal role of EVs in ICP. Those active factors usually consist of lipids, proteins, and nucleotides. The upregulation of miR-21, miR-29a, and miR-590-3p in urinary exosomes can increase the incidence of ICP by downregulating intercellular adhesion molecule 1 (ICAM1) [90]. Nevertheless, more studies are needed to further clarify the role of EVs and EV-encapsulated bioactive factors in ICP.

\subsection{EVS and Spontaneous Abortion or Premature Delivery.} Spontaneous abortion is highly correlated with embryo chromosomal abnormalities. Premature birth will cause great harm to pregnant women and fetuses. The organ development of premature infants is not good. The smaller the gestational age at birth, the lighter the weight, and the worse the prognosis. The causes of premature delivery include infection, malnutrition, cervical insufficiency, uterine malformation, and pregnancy complications. EVs have been shown to be involved in the development of spontaneous abortion and premature delivery by regulating homeostasis imbalances, particularly inflammation and endocrine signals [91]. MSC-derived exosomes regulate maternal-fetal interface $\mathrm{T}$ cells and macrophage-mediated immune response, affecting the outcome of spontaneous abortion or premature delivery [92]. The key role of stem cell-derived EVs in animal models of pregnancy has also been demonstrated [93]. The exact mechanism of endometrial cell-derived EVs mediating maternal crosstalk and affecting pregnancy outcomes during implantation remains unclear. Some studies have shown that EVs derived from placental tissue can predict pregnancy outcomes, including spontaneous abortion and premature delivery. Panfoli et al. confirmed that MSC-EVs affect aerobic metabolism in term and preterm infants [94]. Most importantly, EVs are professional carriers of fetal signals that affect pregnancy outcomes [95]. Amniotic fluid EVs have been shown to deliver information to normal and abnormal births [96]. In addition, exosomal miRNAs were found in maternal circulation, which may represent the "fingerprint" of pregnancy progression [97]. In summary, the study of EVs and their bioactive factors in spontaneous abortion or premature delivery is expected to find new therapeutic strategies for these diseases.

3.6. EVS and Prenatal Diagnosis. Prenatal diagnosis includes chromosomal abnormalities, sexually related genetic diseases, genetic metabolic defects, and congenital structural abnormalities. The important role of circulating EVs has been demonstrated in prenatal and neurodegenerative diseases [98]. Studies have shown that EV miRNAs can be used as biomarkers for prenatal diagnosis of congenital hydronephrosis [99]. They found that reduced expression of EVtransmitting miR-300 and miR-299-5p in amniotic fluid of congenital hydronephrosis could predict renal fibrosis. Interestingly, studies by Goetzl et al. showed that EVs derived from fetal central nervous system could be purified from maternal plasma, which was related to the abnormal proliferation and differentiation of neural stem cells [100]. Therefore, EVs may be a potential way for early prenatal diagnosis of fetal neurological diseases. EVs, especially exosomes, are also involved in Down syndrome [101], suggest- ing that EVs play an important role in the regulation of central nervous system development. Although EVs and the transfer of bioactive factors have been associated with some prenatal and neurodegenerative diseases, more studies need to explore their role in prenatal diagnosis.

\section{Conclusion and Perspectives}

At present, studies have shown that EVs play a key role in tumorigenesis, stem cell capacity maintenance, inflammation, and immune disorders. They participate in the exchange of substances and information between cells by transmitting bioactive molecules, including lipids, proteins, and nucleotides. Cellular EVs in placenta tissue have a good record in maternal circulation, thus affecting pregnancy outcomes. Targeting EVs and the encapsulated bioactive factors may be promising strategies for the treatment and prevention of pregnancy disorders. However, the molecular mechanism and function of EVs in placenta and pregnancy-related complications warrant further elucidation in the future.

\section{Conflicts of Interest}

The authors declare that they have no conflicts of interest.

\section{Authors' Contributions}

Zengfang Wang, Ruizhen Yang, Jiaojiao Zhang, and Pingping Wang contributed equally to this work.

\section{Acknowledgments}

This work is funded by the Weifang Health Science and Technology Program (wfwsjk2019-031) and Shandong Medical and Health Science and Technology Program (2018WS091 and 2015WS0085).

\section{References}

[1] C. Théry, K. W. Witwer, E. Aikawa et al., "Minimal information for studies of extracellular vesicles 2018 (MISEV 2018): a position statement of the International Society for Extracellular Vesicles and update of the MISEV2014 guidelines," Journal of Extracellular Vesicles, vol. 7, no. 1, 2018.

[2] G. van Niel, G. D'Angelo, and G. Raposo, "Shedding light on the cell biology of extracellular vesicles," Nature Reviews. Molecular Cell Biology, vol. 19, no. 4, pp. 213-228, 2018.

[3] M. Colombo, G. Raposo, and C. Thery, "Biogenesis, secretion, and intercellular interactions of exosomes and other extracellular vesicles," Annual Review of Cell and Developmental Biology, vol. 30, no. 1, pp. 255-289, 2014.

[4] J. Meldolesi, "Exosomes and ectosomes in intercellular communication," Current Biology, vol. 28, no. 8, pp. R435R444, 2018.

[5] Q. Qu, Y. Pang, C. Zhang, L. Liu, and Y. Bi, "Exosomes derived from human umbilical cord mesenchymal stem cells inhibit vein graft intimal hyperplasia and accelerate reendothelialization by enhancing endothelial function," Stem Cell Research \& Therapy, vol. 11, no. 1, 2020.

[6] R. M. Johnstone, M. Adam, and B. T. Pan, "The fate of the transferrin receptor during maturation of sheep reticulocytes 
in vitro," Canadian Journal of Biochemistry and Cell Biology, vol. 62, no. 11, pp. 1246-1254, 1984.

[7] H. Valadi, K. Ekström, A. Bossios, M. Sjöstrand, J. J. Lee, and J. O. Lötvall, "Exosome-mediated transfer of mRNAs and microRNAs is a novel mechanism of genetic exchange between cells," Nature Cell Biology, vol. 9, no. 6, pp. 654659, 2007.

[8] G. Lia, C. di Vito, M. Cerrano et al., "Extracellular vesicles after allogeneic hematopoietic cell transplantation: emerging role in post-transplant complications," Frontiers in Immunology, vol. 11, p. 422, 2020.

[9] A. Jurj, O. Zanoaga, C. Braicu et al., “A comprehensive picture of extracellular vesicles and their contents. molecular transfer to cancer cells," Cancers, vol. 12, no. 2, p. 298, 2020.

[10] C. Yang, S. Sun, Q. Zhang et al., "Exosomes of antler mesenchymal stem cells improve postoperative cognitive dysfunction in cardiopulmonary bypass rats through inhibiting the TLR2/TLR4 signaling pathway," Stem Cells International, vol. 2020, Article ID 2134565, 13 pages, 2020.

[11] M. Khan, E. Nickoloff, T. Abramova et al., "Embryonic stem cell-derived exosomes promote endogenous repair mechanisms and enhance cardiac function following myocardial infarction," Circulation Research, vol. 117, no. 1, pp. 52-64, 2015.

[12] G. Zhao, Y. Ge, C. Zhang et al., "Progress of mesenchymal stem cell-derived exosomes in tissue repair," Current Pharmaceutical Design, vol. 26, no. 17, pp. 2022-2037, 2020.

[13] M. Shao, M. Jin, S. Xu et al., "Exosomes from long noncoding RNA-Gm37494-ADSCs repair spinal cord injury via shifting microglial M1/M2 polarization," Inflammation, vol. 43, no. 4, pp. 1536-1547, 2020.

[14] A. Casado-Diaz, J. M. Quesada-Gomez, and G. Dorado, "Extracellular vesicles derived from mesenchymal stem cells (MSC) in regenerative medicine: applications in skin wound healing," Frontiers in Bioengineering and Biotechnology, vol. 8, p. 146, 2020.

[15] C. F. Ruivo, B. Adem, M. Silva, and S. A. Melo, "The biology of cancer exosomes: insights and new perspectives," Cancer Research, vol. 77, no. 23, pp. 6480-6488, 2017.

[16] R. S. Que, C. Lin, G. P. Ding, Z. R. Wu, and L. P. Cao, "Increasing the immune activity of exosomes: the effect of miRNA-depleted exosome proteins on activating dendritic cell/cytokine-induced killer cells against pancreatic cancer," Journal of Zhejiang University. Science. B, vol. 17, no. 5, pp. 352-360, 2016.

[17] F. Fanini and M. Fabbri, "Cancer-derived exosomic microRNAs shape the immune system within the tumor microenvironment: state of the art," Seminars in Cell \& Developmental Biology, vol. 67, pp. 23-28, 2017.

[18] M. G. Appiah, E. J. Park, S. Darkwah et al., "Intestinal epithelium-derived luminally released extracellular vesicles in sepsis exhibit the ability to suppress TNF- $\alpha$ and IL-17A expression in mucosal inflammation," International Journal of Molecular Sciences, vol. 21, no. 22, p. 8445, 2020.

[19] G. Raposo, H. W. Nijman, W. Stoorvogel et al., "B lymphocytes secrete antigen-presenting vesicles," The Journal of Experimental Medicine, vol. 183, no. 3, pp. 1161-1172, 1996.

[20] G. Qiu, G. Zheng, M. Ge et al., "Functional proteins of mesenchymal stem cell-derived extracellular vesicles," Stem Cell Research \& Therapy, vol. 10, no. 1, p. 359, 2019.
[21] N. Edo, K. Kawakami, Y. Fujita et al., "Exosomes expressing thyrotropin receptor attenuate autoantibody-mediated stimulation of cyclic adenosine monophosphate production," Thyroid, vol. 29, no. 7, pp. 1012-1017, 2019.

[22] S. Baskaran, M. K. Panner Selvam, and A. Agarwal, "Exosomes of male reproduction," Advances in Clinical Chemistry, vol. 95, pp. 149-163, 2020.

[23] A. Sabapatha, C. Gercel-Taylor, and D. D. Taylor, "Specific isolation of placenta-derived exosomes from the circulation of pregnant women and their immunoregulatory consequences," American Journal of Reproductive Immunology, vol. 56, no. 5-6, pp. 345-355, 2006.

[24] L. Frängsmyr, V. Baranov, O. Nagaeva, U. Stendahl, L. Kjellberg, and L. Mincheva-Nilsson, "Cytoplasmic microvesicular form of Fas ligand in human early placenta: switching the tissue immune privilege hypothesis from cellular to vesicular level," Molecular Human Reproduction, vol. 11, no. 1, pp. 35-41, 2005.

[25] M. Hedlund, A.-C. Stenqvist, O. Nagaeva et al., "Human placenta expresses and secretes NKG2D ligands via exosomes that down-modulate the cognate receptor expression: evidence for immunosuppressive function," Journal of Immunology, vol. 183, no. 1, pp. 340-351, 2009.

[26] M. Gill, C. Motta-Mejia, N. Kandzija et al., "Placental syncytiotrophoblast-derived extracellular vesicles carry active NEP (neprilysin) and are increased in preeclampsia," Hypertension, vol. 73, no. 5, pp. 1112-1119, 2019.

[27] S. A. Mathew, C. Naik, P. A. Cahill, and R. R. Bhonde, "Placental mesenchymal stromal cells as an alternative tool for therapeutic angiogenesis," Cellular and Molecular Life Sciences, vol. 77, no. 2, pp. 253-265, 2020.

[28] X. Li, L. Liu, J. Yang et al., "Exosome derived from human umbilical cord mesenchymal stem cell mediates MiR-181c attenuating burn-induced excessive inflammation," eBioMedicine, vol. 8, pp. 72-82, 2016.

[29] D. Surico, V. Bordino, V. Cantaluppi et al., "Preeclampsia and intrauterine growth restriction: role of human umbilical cord mesenchymal stem cells-trophoblast cross-talk," PLoS One, vol. 14, no. 6, p. e0218437, 2019.

[30] S. Suvakov, H. Cubro, W. M. White et al., "Targeting senescence improves angiogenic potential of adipose-derived mesenchymal stem cells in patients with preeclampsia," Biology of Sex Differences, vol. 10, no. 1, p. 49, 2019.

[31] L. L. Wang, Y. Yu, H. B. Guan, and C. Qiao, "Effect of human umbilical cord mesenchymal stem cell transplantation in a rat model of preeclampsia," Reproductive Sciences, vol. 23, no. 8, pp. 1058-1070, 2016.

[32] S. L. Nguyen, J. W. Greenberg, H. Wang, B. W. Collaer, J. Wang, and M. G. Petroff, "Quantifying murine placental extracellular vesicles across gestation and in preterm birth data with tidyNano: a computational framework for analyzing and visualizing nanoparticle data in R," PLoS One, vol. 14, no. 6, article e0218270, 2019.

[33] S. Sarker, K. Scholz-Romero, A. Perez et al., "Placentaderived exosomes continuously increase in maternal circulation over the first trimester of pregnancy," Journal of Translational Medicine, vol. 12, no. 1, p. 204, 2014.

[34] D. Tannetta, I. Masliukaite, M. Vatish, C. Redman, and I. Sargent, "Update of syncytiotrophoblast derived extracellular vesicles in normal pregnancy and preeclampsia," Journal of Reproductive Immunology, vol. 119, pp. 98-106, 2017. 
[35] D. I. Chiarello, R. Salsoso, F. Toledo, A. Mate, C. M. Vázquez, and L. Sobrevia, "Foetoplacental communication via extracellular vesicles in normal pregnancy and preeclampsia," Molecular Aspects of Medicine, vol. 60, pp. 69-80, 2018.

[36] G. E. Rice, K. Scholz-Romero, E. Sweeney et al., "The effect of glucose on the release and bioactivity of exosomes from first trimester trophoblast cells," The Journal of Clinical Endocrinology and Metabolism, vol. 100, no. 10, pp. E1280-E1288, 2015.

[37] A. G. Lokossou, C. Toudic, P. T. Nguyen et al., "Endogenous retrovirus-encoded Syncytin-2 contributes to exosomemediated immunosuppression of T cells," Biology of Reproduction, vol. 102, no. 1, pp. 185-198, 2020.

[38] S. Sheller-Miller, K. Choi, C. Choi, and R. Menon, "Cyclicrecombinase-reporter mouse model to determine exosome communication and function during pregnancy," American Journal of Obstetrics and Gynecology, vol. 221, no. 5, pp. 502.e1-502.e12, 2019.

[39] F. Pouresmaeili, I. Azari, S. Arsang-Jang, M. Taheri, and S. Ghafouri-Fard, "Association between expression of long noncoding RNAs in placenta and pregnancy features," Personalized Medicine, vol. 16, no. 6, pp. 457-466, 2019.

[40] A. Kamrani, I. Alipourfard, H. Ahmadi-Khiavi et al., "The role of epigenetic changes in preeclampsia," BioFactors, vol. 45, no. 5, pp. 712-724, 2019.

[41] D. Wu, Y. Xu, Y. Zou et al., "Long noncoding RNA 00473 is involved in preeclampsia by LSD1 binding-regulated TFPI2 transcription in trophoblast cells," Molecular Therapy Nucleic Acids, vol. 12, pp. 381-392, 2018.

[42] E. Delorme-Axford, A. Bayer, Y. Sadovsky, and C. B. Coyne, "Autophagy as a mechanism of antiviral defense at the maternal-fetal interface," Autophagy, vol. 9, no. 12, pp. 2173-2174, 2013.

[43] B. Zhang, R. Liang, M. Zheng, L. Cai, and X. Fan, "Surfacefunctionalized nanoparticles as efficient tools in targeted therapy of pregnancy complications," International Journal of Molecular Sciences, vol. 20, no. 15, p. 3642, 2019.

[44] J. E. Song, S. J. Park, K. Y. Lee, and W. J. Lee, “Amniotic fluid HIF $1 \alpha$ and exosomal HIF $1 \alpha$ in cervical insufficiency patients with physical examination-indicated cerclage," The Journal of Maternal-Fetal \& Neonatal Medicine, vol. 32, no. 14, pp. 2287-2294, 2019.

[45] B. Lee, I. Saadeldin, and H. J. Oh, "Embryonic\&ndash;maternal cross-talk via exosomes: potential implications," Stem Cells and Cloning: Advances and Applications, vol. 8, pp. 103-107, 2015.

[46] G. Truong, D. Guanzon, V. Kinhal et al., "Oxygen tension regulates the miRNA profile and bioactivity of exosomes released from extravillous trophoblast cells - liquid biopsies for monitoring complications of pregnancy," PLoS One, vol. 12, no. 3, article e0174514, 2017.

[47] P. Pillay, K. Moodley, J. Moodley, and I. Mackraj, "Placentaderived exosomes: potential biomarkers of preeclampsia," International Journal of Nanomedicine, vol. Volume 12, pp. 8009-8023, 2017.

[48] R. Gadde, D. Cd, and S. R. Sheela, "Placental protein 13: an important biological protein in preeclampsia," Journal of Circulating Biomarkers, vol. 7, article 1849454418786159, 2018.

[49] C. Salomon, D. Guanzon, K. Scholz-Romero et al., "Placental exosomes as early biomarker of preeclampsia: potential role of exosomal microRNAs across gestation," The Journal of
Clinical Endocrinology and Metabolism, vol. 102, no. 9, pp. 3182-3194, 2017.

[50] P. Pillay, N. Maharaj, J. Moodley, and I. Mackraj, "Placental exosomes and pre-eclampsia: maternal circulating levels in normal pregnancies and, early and late onset pre-eclamptic pregnancies," Placenta, vol. 46, pp. 18-25, 2016.

[51] X. Chang, J. Yao, Q. He, M. Liu, T. Duan, and K. Wang, "Exosomes from women with preeclampsia induced vascular dysfunction by delivering sFlt (soluble Fms-like tyrosine kinase)1 and sEng (soluble endoglin) to endothelial cells," Hypertension, vol. 72, no. 6, pp. 1381-1390, 2018.

[52] K. Maduray, J. Moodley, and I. Mackraj, “The impact of circulating exosomes derived from early and late onset preeclamptic pregnancies on inflammatory cytokine secretion by BeWo cells," European Journal of Obstetrics, Gynecology, and Reproductive Biology, vol. 247, pp. 156-162, 2020.

[53] O. Biro and J. Rigo Jr., "The pathogenetic role and expression profile of microRNAs in preeclampsia," Orvosi Hetilap, vol. 159, no. 14, pp. 547-556, 2018.

[54] E. Devor, D. Santillan, S. Scroggins, A. Warrier, and M. Santillan, "Trimester-specific plasma exosome microRNA expression profiles in preeclampsia," The Journal of Maternal-Fetal \& Neonatal Medicine, vol. 33, no. 18, pp. 3116-3124, 2020.

[55] P. Pillay, M. Vatish, R. Duarte, J. Moodley, and I. Mackraj, "Exosomal microRNA profiling in early and late onset preeclamptic pregnant women reflects pathophysiology," International Journal of Nanomedicine, vol. Volume 14, pp. 5637-5657, 2019.

[56] H. Li, Y. Ouyang, E. Sadovsky, W. T. Parks, T. Chu, and Y. Sadovsky, "Unique microRNA signals in plasma exosomes from pregnancies complicated by preeclampsia," Hypertension, vol. 75, no. 3, pp. 762-771, 2020.

[57] O. Biró, B. Alasztics, A. Molvarec, J. Joó, B. Nagy, and J. Rigó Jr., "Various levels of circulating exosomal total-miRNA and miR-210 hypoxamiR in different forms of pregnancy hypertension," Pregnancy Hypertension, vol. 10, pp. 207-212, 2017.

[58] L. Shen, Y. Li, R. Li et al., "Placenta-associated serum exosomal miR-155 derived from patients with preeclampsia inhibits eNOS expression in human umbilical vein endothelial cells," International Journal of Molecular Medicine, vol. 41, no. 3, pp. 1731-1739, 2018.

[59] T. M. K. Motawi, D. Sabry, N. W. Maurice, and S. M. Rizk, "Role of mesenchymal stem cells exosomes derived microRNAs; miR-136, miR-494 and miR-495 in pre-eclampsia diagnosis and evaluation," Archives of Biochemistry and Biophysics, vol. 659, pp. 13-21, 2018.

[60] O. Biró, Á. Fóthi, B. Alasztics, B. Nagy, T. I. Orbán, and J. Rigó Jr., "Circulating exosomal and Argonaute-bound microRNAs in preeclampsia," Gene, vol. 692, pp. 138-144, 2019.

[61] I. Hromadnikova, L. Dvorakova, K. Kotlabova, and L. Krofta, "The prediction of gestational hypertension, preeclampsia and fetal growth restriction via the first trimester screening of plasma exosomal C19MC microRNAs," International Journal of Molecular Sciences, vol. 20, no. 12, p. 2972, 2019.

[62] Z. Xueya, L. Yamei, C. Sha et al., "Exosomal encapsulation of miR-125a-5p inhibited trophoblast cell migration and proliferation by regulating the expression of VEGFA in preeclampsia," Biochemical and Biophysical Research Communications, vol. 525, no. 3, pp. 646-653, 2020. 
[63] Z. H. Xiong, J. Wei, M. Q. Lu, M. Y. Jin, and H. L. Geng, "Protective effect of human umbilical cord mesenchymal stem cell exosomes on preserving the morphology and angiogenesis of placenta in rats with preeclampsia," Biomedicine \& Pharmacotherapy, vol. 105, pp. 1240-1247, 2018.

[64] Y. Chen, H. Ding, M. Wei et al., "MSC-secreted exosomal H19 promotes trophoblast cell invasion and migration by downregulating let-7b and upregulating FOXO1," Molecular Therapy - Nucleic Acids, vol. 19, pp. 1237-1249, 2020.

[65] M. Arias, L. J. Monteiro, S. Acuña-Gallardo et al., "Extracellular vesicle concentration in maternal plasma as an early marker of gestational diabetes," Revista Médica de Chile, vol. 147, no. 12, pp. 1503-1509, 2019.

[66] R. Sharma, M. Kumari, P. Prakash, S. Gupta, and S. Tiwari, "Phosphoenolpyruvate carboxykinase in urine exosomes reflect impairment in renal gluconeogenesis in early insulin resistance and diabetes," American Journal of Physiology. Renal Physiology, vol. 318, no. 3, pp. F720-F731, 2020.

[67] T. Delong, T. A. Wiles, R. L. Baker et al., "Pathogenic CD4 T cells in type 1 diabetes recognize epitopes formed by peptide fusion," Science, vol. 351, no. 6274, pp. 711-714, 2016.

[68] Y. Lytvyn, F. Xiao, C. R. J. Kennedy et al., "Assessment of urinary microparticles in normotensive patients with type 1 diabetes," Diabetologia, vol. 60, no. 3, pp. 581-584, 2017.

[69] A. Sakurai, H. Ono, A. Ochi et al., "Involvement of Elf 3 on Smad 3 activation-dependent injuries in podocytes and excretion of urinary exosome in diabetic nephropathy," PLoS One, vol. 14, no. 5, article e0216788, 2019.

[70] J. Hu, S. Wang, Z. Xiong et al., "Exosomal Mst1 transfer from cardiac microvascular endothelial cells to cardiomyocytes deteriorates diabetic cardiomyopathy," Biochimica et Biophysica Acta - Molecular Basis of Disease, vol. 1864, no. 11, pp. 3639-3649, 2018.

[71] D. Beuzelin and B. Kaeffer, "Exosomes and miRNA-Loaded biomimetic nanovehicles, a focus on their potentials preventing type-2 diabetes linked to metabolic syndrome," Front Immunol, vol. 9, p. 2711, 2018.

[72] P. Venkat, C. Cui, Z. Chen et al., "CD133+exosome treatment improves cardiac function after stroke in type 2 diabetic mice," Translational Stroke Research, vol. 12, pp. 112-124, 2020.

[73] J. Chen, J. Chen, Y. Cheng et al., "Mesenchymal stem cellderived exosomes protect beta cells against hypoxia-induced apoptosis via miR-21 by alleviating ER stress and inhibiting p38 MAPK phosphorylation," Stem Cell Research \& Therapy, vol. 11, no. 1, p. 97, 2020.

[74] P. Venkat, M. Chopp, and J. Chen, "Cell-based and exosome therapy in diabetic stroke," Stem Cells Translational Medicine, vol. 7, no. 6, pp. 451-455, 2018.

[75] J. Liu, S. Z. Wang, Q. L. Wang, J. G. Du, and B. B. Wang, "Gestational diabetes mellitus is associated with changes in the concentration and bioactivity of placental exosomes in the maternal circulation across gestation," European Review for Medical and Pharmacological Sciences, vol. 22, no. 7, pp. 2036-2043, 2018.

[76] T. Saez, P. de Vos, L. Sobrevia, and M. M. Faas, "Is there a role for exosomes in foetoplacental endothelial dysfunction in gestational diabetes mellitus?," Placenta, vol. 61, pp. 48-54, 2018.

[77] N. Jayabalan, A. Lai, V. Ormazabal et al., "Adipose tissue exosomal proteomic profile reveals a role on placenta glucose metabolism in gestational diabetes mellitus," The Journal of Clinical Endocrinology and Metabolism, vol. 104, no. 5, pp. 1735-1752, 2019.

[78] L. B. James-Allan, F. J. Rosario, K. Barner et al., "Regulation of glucose homeostasis by small extracellular vesicles in normal pregnancy and in gestational diabetes," The FASEB Journal, vol. 34, no. 4, pp. 5724-5739, 2020.

[79] E. Guarino, C. Delli Poggi, G. E. Grieco et al., "Circulating MicroRNAs as Biomarkers of Gestational Diabetes Mellitus: Updates and Perspectives," International Journal of Endocrinology, vol. 2018, Article ID 6380463, 11 pages, 2018.

[80] S. Nair, N. Jayabalan, D. Guanzon et al., "Human placental exosomes in gestational diabetes mellitus carry a specific set of miRNAs associated with skeletal muscle insulin sensitivity," Clinical Science (London, England), vol. 132, no. 22, pp. 2451-2467, 2018.

[81] J. F. Floriano, G. Willis, F. Catapano et al., "Exosomes could offer new options to combat the long-term complications inflicted by gestational diabetes mellitus," Cells, vol. 9, no. 3, p. $675,2020$.

[82] A. S. Herrera-Van Oostdam, M. Salgado-Bustamante, J. A. Lopez, D. A. Herrera-Van Oostdam, and Y. Lopez-Hernandez, "Placental exosomes viewed from an 'omics' perspective: implications for gestational diabetes biomarkers identification," Biomarkers in Medicine, vol. 13, no. 8, pp. 675-684, 2019.

[83] Q. Ge, Y. Zhu, H. Li, F. Tian, X. Xie, and Y. Bai, "Differential expression of circulating miRNAs in maternal plasma in pregnancies with fetal macrosomia," International Journal of Molecular Medicine, vol. 35, no. 1, pp. 81-91, 2015.

[84] R. S. Rodosthenous, H. H. Burris, A. P. Sanders et al., "Second trimester extracellular microRNAs in maternal blood and fetal growth: an exploratory study," Epigenetics, vol. 12, no. 9, pp. 804-810, 2017.

[85] J. Miranda, C. Paules, S. Nair et al., "Placental exosomes profile in maternal and fetal circulation in intrauterine growth restriction - liquid biopsies to monitoring fetal growth," Placenta, vol. 64, pp. 34-43, 2018.

[86] J. Luo, Y. Fan, L. Shen et al., "The pro-angiogenesis of exosomes derived from umbilical cord blood of intrauterine growth restriction pigs was repressed associated with MiRNAs," International Journal of Biological Sciences, vol. 14, no. 11, pp. 1426-1436, 2018.

[87] I. Hromadnikova, K. Kotlabova, M. Ondrackova et al., "Expression profile of C19MC microRNAs in placental tissue in pregnancy-related complications," DNA and Cell Biology, vol. 34 , no. 6, pp. 437-457, 2015.

[88] K. Kotlabova, J. Doucha, and I. Hromadnikova, "Placentalspecific microRNA in maternal circulation - identification of appropriate pregnancy-associated microRNAs with diagnostic potential," Journal of Reproductive Immunology, vol. 89, no. 2, pp. 185-191, 2011.

[89] I. Hromadnikova, K. Kotlabova, K. Ivankova, and L. Krofta, "First trimester screening of circulating C19MC microRNAs and the evaluation of their potential to predict the onset of preeclampsia and IUGR," PLoS One, vol. 12, no. 2, article e0171756, 2017.

[90] P. Y. Jiang, X. J. Zhu, R. A. Jiang, Y. N. Zhang, L. Liu, and X. F. Yang, "MicroRNAs derived from urinary exosomes act as novel biomarkers in the diagnosis of intrahepatic cholestasis of pregnancy," American Journal of Translational Research, vol. 11, no. 9, pp. 6249-6261, 2019. 
[91] N. Jayabalan, A. Lai, S. Nair et al., "Quantitative proteomics by SWATH-MS suggest an association between circulating exosomes and maternal metabolic changes in gestational diabetes mellitus," Proteomics, vol. 19, no. 1-2, article e1800164, 2019.

[92] Y. J. Xiang, Y. Y. Hou, H. L. Yan et al., "Mesenchymal stem cells-derived exosomes improve pregnancy outcome through inducing maternal tolerance to the allogeneic fetus in abortion-prone mating mouse," Kaohsiung Journal of Medical Sciences, vol. 36, no. 5, pp. 363-370, 2020.

[93] A. Mitchell, H. Wanczyk, T. Jensen, and C. Finck, "Human induced pluripotent stem cells ameliorate hyperoxiainduced lung injury in a mouse model," American Journal of Translational Research, vol. 12, no. 1, pp. 292-307, 2020.

[94] I. Panfoli, S. Ravera, M. Podestà et al., "Exosomes from human mesenchymal stem cells conduct aerobic metabolism in term and preterm newborn infants," The FASEB Journal, vol. 30, no. 4, pp. 1416-1424, 2016.

[95] C. Salomon, Z. Nuzhat, C. L. Dixon, and R. Menon, "Placental exosomes during gestation: liquid biopsies carrying signals for the regulation of human parturition," Current Pharmaceutical Design, vol. 24, no. 9, pp. 974-982, 2018.

[96] C. L. Dixon, S. Sheller-Miller, G. R. Saade et al., "Amniotic fluid exosome proteomic profile exhibits unique pathways of term and preterm labor," Endocrinology, vol. 159, no. 5, pp. 2229-2240, 2018.

[97] R. Menon, C. Debnath, A. Lai et al., "Circulating exosomal miRNA profile during term and preterm birth pregnancies: a longitudinal study," Endocrinology, vol. 160, no. 2, pp. 249-275, 2019.

[98] X. Cai, F. Janku, Q. Zhan, and J. B. Fan, “Accessing genetic information with liquid biopsies," Trends in Genetics, vol. 31, no. 10, pp. 564-575, 2015.

[99] J. Xie, Y. Zhou, W. Gao, Z. Li, Z. Xu, and L. Zhou, "The relationship between amniotic fluid miRNAs and congenital obstructive nephropathy," American Journal of Translational Research, vol. 9, no. 4, pp. 1754-1763, 2017.

[100] L. Goetzl, N. Darbinian, and N. Merabova, "Noninvasive assessment of fetal central nervous system insult: potential application to prenatal diagnosis," Prenatal Diagnosis, vol. 39, no. 8, pp. 609-615, 2019.

[101] E. D. Hamlett, A. LaRosa, E. J. Mufson, J. Fortea, A. Ledreux, and A. C. Granholm, "Exosome release and cargo in Down syndrome," Developmental Neurobiology, vol. 79, no. 7, pp. 639-655, 2019. 